\title{
Obstetrics at Decisive Crossroads Regarding Pattern- Recognition of Fetal Heart Rate Decelerations: Scientific Principles and Lessons From Memetics
}

\author{
Shashikant L. Sholapurkar
}

\begin{abstract}
The survival of cardiotocography (CTG) as a tool for intrapartum fetal monitoring seems threatened somewhat unjustifiably and unwittingly despite the absence of better alternatives. Fetal heart rate (FHR) decelerations are center-stage (most important) in the interpretation of CTG with maximum impact on three-tier classification. The pattern-discrimination of FHR decelerations is inexorably linked to their nomenclature. Unscientific or flawed nomenclature of decelerations can explain the dysfunctional CTG interpretation leading to errors in detection of acidemic fetuses. There are three contrasting concepts about categorization of FHR decelerations: 1) all rapid decelerations (the vast majority) should be grouped as "variable" because they are predominantly due to cord-compression, 2) all decelerations are due to chemoreflex from fetal hypoxemia hence their timing is not important, and 3) FHR decelerations should be categorized into "early/late/variable" based primarily on their time relationship to contractions. These theoretical concepts are like memes (ideas/beliefs). Lessons from "memetics" are that the most popular, attractive or established beliefs may not necessarily be true, scientific, beneficial or even without harm. Decelerations coincident with contractions with trough corresponding to the peak of contractions cannot be explained by cord-compression or increasing hypoxia (from compromised uteroplacental perfusion, cord-compression or even cerebral hypoperfusion/anoxia purportedly conceivable from headcompression). Decelerations due to hypoxemia would be associated with delayed recovery of decelerations (lag phase). It is a scientific imperative to cast away disproven/falsified theories. Practices based on unscientific theories lead to patient harm. Clinicians should urgently adopt the categorization of FHR decelerations based primarily of the time relationship to contractions as originally proposed by Hon and Caldeyro-Barcia. This analytical review shows it to be underpinned by most robust physiological and scientific hypotheses unlike the other categorizations associated with untruthful hypotheses, irreconcilable fallacies and contradictions. Without truthful framework and meaningful pattern-recognition of FHR decelerations, the CTG will not fulfil its true potential.
\end{abstract}

Manuscript submitted December 8, 2017, accepted December 20, 2017

Department of Obstetrics and Gynaecology, Royal United Hospitals Bath NHS Foundation Trust, Combe Park, Bath, UK. Email: s.sholapurkar@nhs.net

doi: https://doi.org/10.14740/jocmr3307e
Keywords: Cardiotocography; Fetal heart rate decelerations; Memetics; Intrapartum fetal monitoring; Head compression; Cord compression; Fetal hypoxemia

\section{Introduction}

Fetal asphyxia in labor has devastating consequences. Cardiotocography (CTG) has been found wanting in detecting fetal acidemia [1-6] but would continue to be practiced widely because the alternatives like fetal ECG (ST analysis) or fetal oximetry remain unproven [6]. Hence, it seems very important to identify areas for improvement or reform of CTG interpretation. All guidelines concur on definitions of fetal heart rate (FHR) baseline, variability and accelerations [4-7]. However, they differ significantly on interpretation of FHR decelerations which happen to be center stage and critically important in the pattern-recognition of CTG [8]. When a non-acidemic fetus develops serious acidemia during labor, it invariably displays FHR decelerations reflecting or giving vital clues to its deterioration. On the other hand, common experience shows that the majority of FHR decelerations do not lead to fetal acidemia and hence many are likely to be of non-hypoxemic etiology. If we do not interpret decelerations scientifically, there is little hope of interpreting CTG correctly. The pathophysiology and interpretation of FHR decelerations have become very contested in the last decade although there was little controversy in British practice before 2007 [9]. Obstetrics internationally has arrived at crossroads with three main paths to choose in this most vital aspect, viz. 1) all rapid decelerations (the vast majority) are due to cord-compression and hence must be called "variable", 2) all decelerations are due to chemoreflex from fetal asphyxia (or hypoxemia) and their time relationship to contractions is irrelevant [10], 3) FHR decelerations should be classified into "early/late/variable" based primarily on their time relationship to the contractions as proposed by pioneers like Hon and Caldeyro-Barcia $[9,11,12]$. Only one of the three approaches must be true or correct. But does the truth matter? Although FHR decelerations are center-stage, is their categorization/nomenclature at all important? The principle objective of this short article is to highlight the critical importance of adopting a reformed scientific categorization of FHR decelerations if the visual CTG 
interpretation is to survive as a clinically useful technique. This article also draws analogy from "memetics" as to how different opinions develop, disseminate and persist.

\section{Memetics}

An internationally renowned scientist Richard Dawkins in his acclaimed 1976 book "The Selfish Gene" proposed "memetics" as a theory of "mental content" based on an analogy with Darwinian evolution. The "meme" (analogous to a "gene") was conceived as a unit of culture (an idea, belief, narrative, pattern of behaviour etc.) which is "hosted" in the minds of one or more individuals and which can reproduce itself by jumping from mind to mind [13]. The Oxford Dictionary defines memes as "replicators: tunes, ideas, catch-phrases, clothes fashions etc.". Memetics is notable for sidestepping the traditional concern with the truth of ideas and beliefs. Instead, it is interested in their success. A meme's success may be due to its contribution to the effectiveness of its host [13]. Thus the meme and the host may develop a mutually beneficial relationship. Moreover, ideas (memes) can come to control thinking and people themselves. Even more revolutionary is a concept that memes like genes can spread even if the host may suffer in the process (extreme examples are kamikaze pilots and more recently suicide-bombers) [13]. Memes are replicators i.e. copied by imitation, teaching and other methods. The copies are not perfect: memes are copied with variation; moreover, they compete for space in our memories and for the chance to be copied again. Only some of the variants can survive. The combination of these three elements (copies, variation and competition for survival) forms precisely the condition for Darwinian evolution, and so memes (and hence human beliefs and cultures) evolve. Large groups of memes that are copied and passed on together are called "memeplexes" [13]. The Center for Policy Modelling at Manchester Metropolitan University hosted an e-journal entitled the "Journal of Memetics-Evolutionary Models of Information Transmission" on the web and there has been short lived paper-based memetics publication the "Journal of Ideas" starting in 1990 [13]. Do principles of memetics apply to scientific ideas? Science should be concerned with facts and truths rather than attractiveness, popularity or chance-survival of ideas. Medicine is an applied rather than a pure science and hence memetics would have some place in it, a major one in medico-politics. One could consider the ideas and hypotheses about FHR decelerations as "memeplexes".

\section{Critical Importance of FHR Decelerations in Three-Tier Systems}

The three-tier systems of CTG interpretation take into account different gradations of many FHR parameters (baseline, variability, accelerations and decelerations) and arrive at a judgement viz. normal/suspicious/pathological CTG. The American three-tier system has been found clinically unhelpful [2, 14] because its pathological (grade III) category has been designed to correlate with severe/dangerous fetal acidemia rather than lesser levels of acidemia where diagnosis and clinical intervention need to be targeted. Another weakness seems applying the same depth-duration criteria for FHR decelerations in high/low risk cases and in the first and second stages of labor [2]. Sometimes there is too much focus on the specific cut-offs of the FHR parameters or what combinations should warrant which tier. There seems nothing definitive/sacrosanct about these. The three-tier systems function by the macro-amalgamation of multiple parameters with varying weightage erring on the side of high sensitivity to detect moderate fetal acidemia. FHR decelerations are the most common aberrations with maximum impact on classification of CTG in the three-tier system. Thus, rather than the variations within the three-tier systems, the interpretation/categorization of FHR decelerations seems far more crucial [9]. It is important to note that although loss of baseline variability has strong correlation with severe acidemia $(\mathrm{pH}<7.00)$ and hypoxic encephalopathy; it is seen in only a very small percentage of fetuses with moderate acidemia ( $\mathrm{pH}<7.10)$, where obstetric intervention needs to be targeted $[15,16]$. Hence, more often the decision to intervene needs to be based on interpretation FHR decelerations alone (category II of three-tier American system) mostly without baseline changes.

\section{Etiological Versus Pathophysiological Categori- zation of FHR Decelerations}

The precise etiology of FHR decelerations in human labor in individual cases will mostly remain presumptive. Hence, for clinical application, the decelerations are best classified based on hypoxic or non-hypoxic nature rather than presumptive causation, the latter has caused confusion and contradictions. In the last decade it has become common to suggest that vast majority of decelerations (rapid descent) are because of cordcompression and hence must be called "variable" [5-7]. This has been mainly a result of over-simplistic adoption of observations from animal studies involving cord-occlusion which showed rapid decelerations [14]. Another (mis)belief has been that if decelerations vary in depth or width, they should be called variable [9]. But that will include all decelerations, thus an antithesis of classification. Experiments by Edward Hon in human labor with pressure on fetal head with ring pessaries introduced in vagina showed rapid short FHR decelerations lasting for $15 \mathrm{~s}$ or so [17]. Moreover, the pioneering group of Caldeyro-Barcia showed a strong empirical evidence in human labor that repeated manual head compressions mimicking that caused by contractions produced decelerations lasting for $30 \mathrm{~s}$ or so very similar to rapid type 1 (early) (Figs. 1 and 2) [12]. This disproves the theory that severe cerebral hypoperfusion and unsafe hypoxia are necessary for head-compression to cause FHR decelerations [10], a belief also completely contradictory to the actual clinical experience of most obstetricians. Causation of FHR decelerations is likely to be multifactorial with head-compression making some contribution. More importantly, rather than presumptive etiology, what seems clinically most relevant is to analyse whether the characteristics/ 


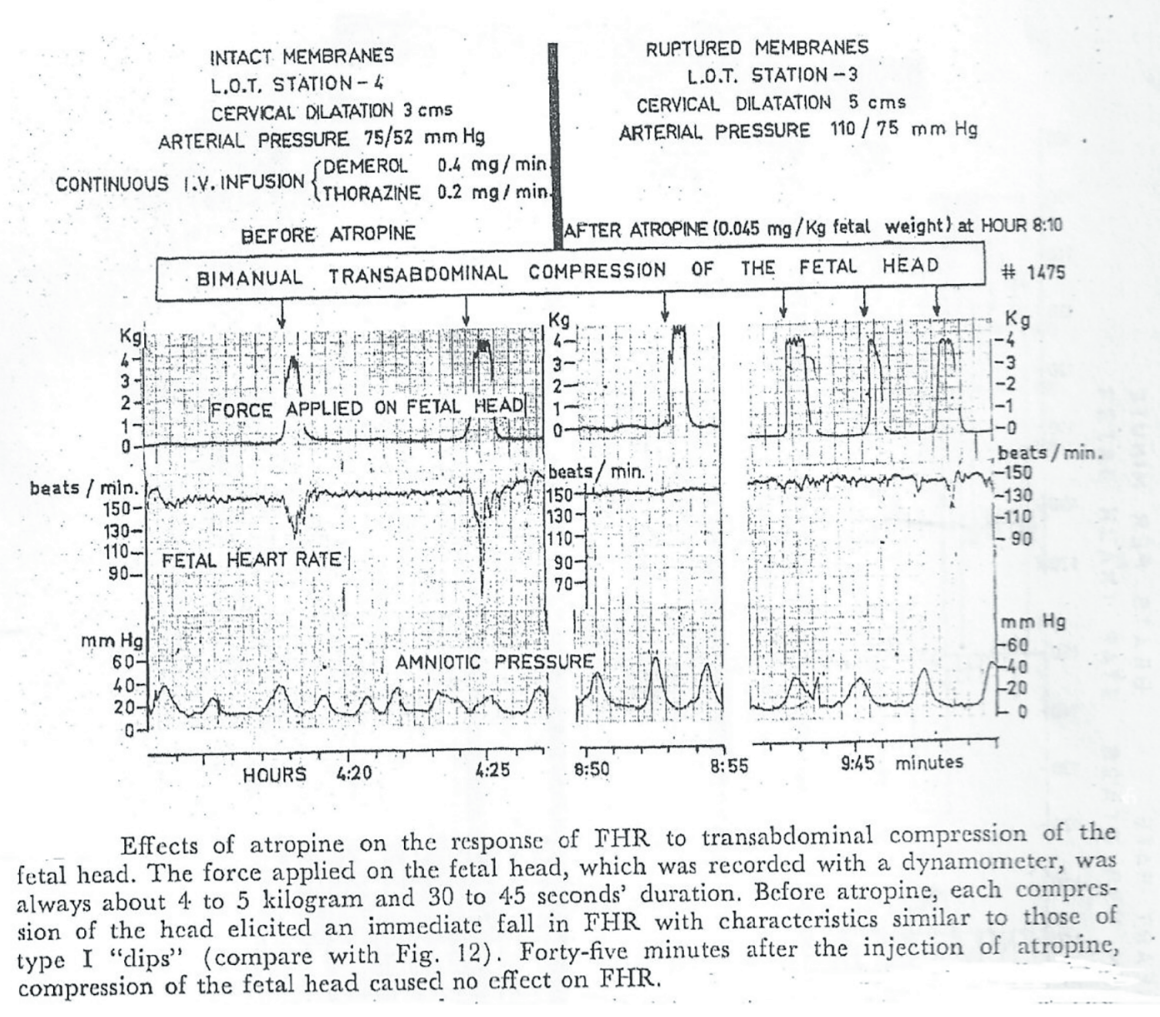

Figure 1. Direct empirical evidence showing transabdominal pressure on fetal head causes short lasting rapid FHR decelerations which are suppressed after atropine administration in human labor (gratefully reproduced with kind permission from MendezBauer et al [12])

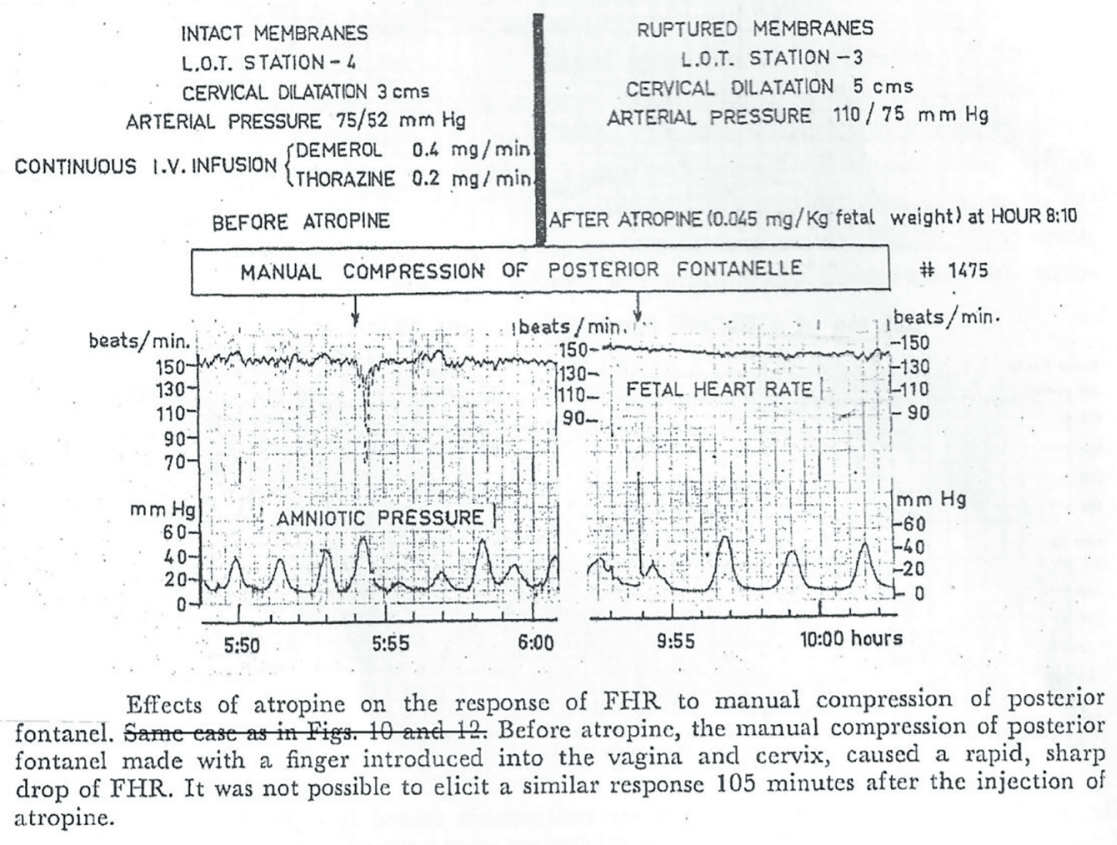

Figure 2. Direct empirical evidence showing pressure on posterior fontanelle with a finger introduced in vagina causes short lasting rapid FHR decelerations which are suppressed after atropine administration in human labor (gratefully reproduced with kind permission from Mendez-Bauer et al [12]). 

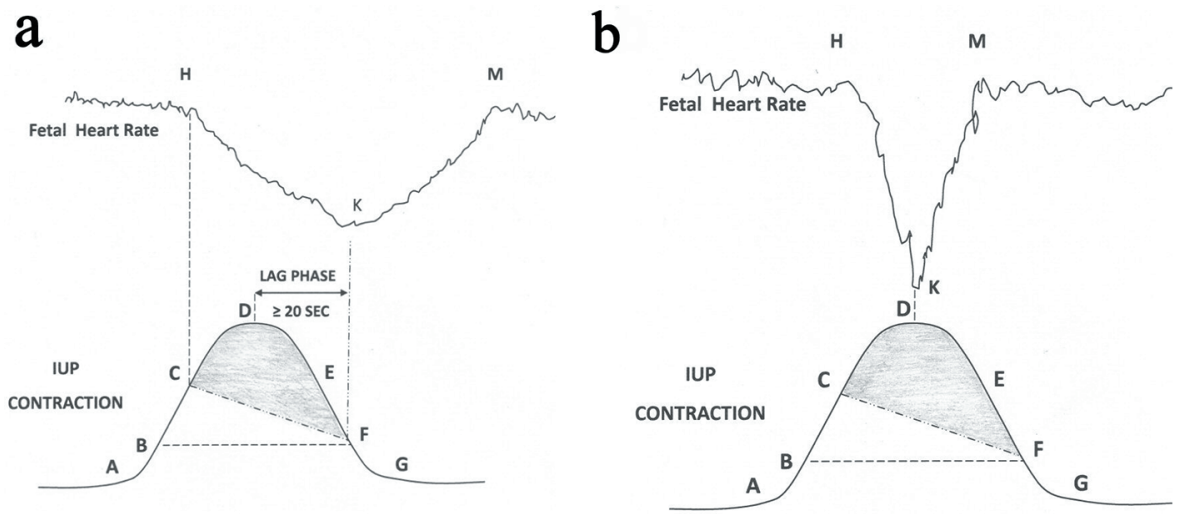

Figure 3. (a) Schematic drawing of FHR deceleration resulting from peripheral chemoreflex due to hypoxemia based on scientific rationale. Hypoxemic trigger is very likely to produce a classical "late deceleration" [9]. Shaded area: level of IUP where fetal $\mathrm{PaO}_{2}$ will continue to drop during deceleration; Point A: contraction commences; $\mathrm{B}$ : IUP enough to commence fetal hypoxemia; C: worsening fetal hypoxemia enough to start FHR deceleration; D: peak of contraction where speed of worsening of hypoxemia will slow down but hypoxemia will continue to worsen $\left(\mathrm{PaO}_{2}\right.$ continues to drop); $\mathrm{E}$ : hypoxemia will continue to worsen; $\mathrm{F}$ : hypoxia will start recovering because IUP equivalent to point $\mathrm{B}$. Chemoreflex induced FHR deceleration will start recovering at point $\mathrm{F}$ and recovery will extend beyond the end of contraction. FHR: fetal heart rate; IUP: intrauterine pressure; $\mathrm{PaO}_{2}$ : fetal partial pressure of oxygen. (b): Schematic drawing showing that the common rapid short-lasting FHR decelerations in labor cannot be explained by fetal hypoxemia.

patterns of different decelerations give clue about hypoxic or non-hypoxic mechanisms underlying them [9]. The majority of decelerations during labor look rapid on British CTG (paper speed $1 \mathrm{~cm} / \mathrm{min}$ ) and coincide with contractions with trough corresponding with peak of contraction and recovering before the end of contraction [9]. Cord-compression has been suggested as underlying mechanism [5-7] with the commonly quoted baroreceptor hypothesis proposing complete cord occlusion for common benign rapid decelerations with "shoulders" $[14,16]$. This seems doubly fallacious. Complete cord-compression cannot simply be underlying cause for vast majority of decelerations in labor. Secondly, chemoreflex in response to hypoxemia is a more dominant reflex during cordcompression than baroreflex $[8,10,14,18]$. Moreover, the hypoxemia (hence the deceleration) will start recovering only after relief of umbilical venous compression much later during the relaxation phase and not around the peak of contraction $[9$, $14,18]$.

Recently, there has been another school of thought that all FHR decelerations during labor are due to hypoxemia from reduction in uteroplacental perfusion (not from cordcompression after all) [10]. Fetal pulse oximetry studies show that there are milder degrees of transient fetal hypoxemia (but not acidemia or asphyxia) during most contractions, but not enough to cause FHR decelerations [10]. Hence, it has been suggested that whenever decelerations occur, they must be because of fetal hypoxemia getting worse [10]. This seems denial of all non-hypoxemic mechanisms. Further conclusion has been drawn that since all decelerations are because of hypoxemia, it is pointless to classify decelerations into "early, late and variable" as their time relationship to (peak of) contractions does not give any information [10, 19]. There are major and irrevocable fallacies with this hypothesis. If fetal hypoxemia (severe enough to start a deceleration) develops during contraction phase, then this hypoxemia will not start recovering at the height of contraction (especially with increasing degrees of tissue hypoxia) but only much later in the relaxation phase (Fig. 3a, b). Thus, decelerations with their trough corresponding to peak of contraction (the majority in labor) simply cannot be explained by "hypoxemic" mechanism (due to drop in uteroplacental perfusion, cord-compression or even fetal cerebral hypoperfusion/anoxia purportedly conceivable from head-compression) as the hypoxemia will continue to worsen up to point F (Fig. 3b). This is also supported by many animal experiments showing that the decelerations start to recover only after relief of cord-occlusion $[18,20]$. Singular select animal experiment showing partial recovery of FHR during prolonged complete cord-occlusion (confirmation bias) [21] cannot explain the consistent recovery of most decelerations corresponding to the peak of contractions and cannot be extrapolated to human labor. These most common decelerations (Fig. 3b) can be explained by non-hypoxemic vagal reflex, possibly multifactorial with head compression playing a role [9]. Moreover, the recovery of hypoxemia (and thus that of the deceleration) is very likely to extend beyond the end of contraction (Fig. 3a). This is in fact the classical concept of "late decelerations" quite rightly defined by RCOG as, "Decelerations with nadir more than 20 $\mathrm{s}$ (lag period) after the peak of contraction and recovery after the end of contraction" [4].

The first two schools of thought heavily depend on denial of head compression as a mechanism for benign decelerations. The architects and primary proponents of both schools have been "experimental physiologists" with no clinical experience of conducting human labor or interpreting CTGs. They often take an absolutist view that head-compression must always or consistently cause decelerations or must explain the deceleration as a sole isolated mechanism. Secondly, they propose that head-compression causes decelerations only with severe compression of head with cerebral ischemia/hypoxemia; 
hence early decelerations are extremely rare [10]. Somewhat misplaced emphasis on designating singular etiology has led to disproportionate importance given to sheep fetus experiments with binary methodology of artificial cord-occlusion or head compression (at the expense of clinical experience). The findings (like cerebral ischemia from head-compression) from the unphysiological animal experiments should not be directly extrapolated to much more complex and multiple factors involved during labor in clinical practice. Head-compression is very common throughout the human labor (unlike sheep) and can even be quite pronounced. Intrapartum fetal neurological injury due to cerebral anoxia due to head-compression in the absence of systemic hypoxemia-acidemia simply does not seem to be a notable concern in the clinical practice and guidelines [5-7, 22] despite a century of intrapartum fetal monitoring, probably for sound reasons. The evidence available on the effect of head compression on intracranial pressure, oxygenation, blood flow and cerebral function does not support the intrapartum fetal extracranial pressure as a cause of fetal brain injury, although it remains a popular theory in legal circles [23]. Moreover, this is contrary to the best possible and direct empirical evidence in human labor (Figs. 1 and 2) $[12,17]$. Importantly, most birth attendants have observed head-compression causing short lasting decelerations and their experience and their intuition (meme) is most likely right than the contrary meme. Particularly interesting is a common observation even mentioned by Hon that during twin labor the first twin with cephalic presentation far more frequently displays rapid decelerations with early timing before and more commonly after rupture of membranes [9]. These can be best explained by head-compression only because both twins are equally exposed to hypoxemic effects of drop in uteroplacental circulation or cord-compression [9]. The precise mechanism of head-compression decelerations is unknown but vagal stimulation due to distortion of brain envelops and transient rise in intracranial pressure could be responsible.

Thus, it seems reasonable to argue that the original categorization of decelerations by Hon and Caldeyro-Barcia based primarily on time relationship to the uterine contraction seems most scientific $[9,11,12]$. This was the system practiced by the British obstetricians and midwives until 2007 and is best revived.

\section{CTG Interpretation: Science Plus Art}

CTG interpretation and decision-making involves analysis of complex FHR patterns in the context of materno-fetal characteristics and status of labor. In addition to the scientific principles, this does involve subjective judgement and intuition: the art. However, this "art" is acquired by experience from practicing scientific concepts and framework. Senior British obstetricians often bemoan that the art of CTG interpretation is being lost but they were fortunate to have had the opportunity to learn the art in the first place by practicing traditional British system before 2007 [9]. The junior obstetricians and midwives, being exposed to unscientific framework of wrong pattern-recognition, would be unable to acquire the art.

\section{Does Nomenclature of FHR Decelerations Mat- ter?}

It is a common presumption that what "label" we give FHR decelerations does not matter as long as we all practice the same standardised framework. However, this seems a misconception because the interpretation of FHR decelerations is inexorably linked to their nomenclature [9]. The current framework does not recognize benign early decelerations (should be more common) which is of course a major handicap. Findings of laboratory animal experiments, unrepresentative of complex multiple factors in human labor, should be interpreted with caution especially when they contradict the long clinical experience. The vast majority of FHR decelerations are (mis) labelled as "variable" due to cord-compression based on hypothesis which is fatally flawed $[9,16]$. A major effort gets misdirected in discriminating these (false) "cord-compression decelerations" using arbitrary, random and varied criteria depending on which guideline one refers to. But the main cause of fetal hypoxemia-acidemia in labor is contraction induced drop in utero-placental perfusion and not cord-compression at all. This distorted and unscientific framework causes falsealarm fatigue, confusion and difficulty in focusing on the smaller number of real pathological decelerations $[9,16]$. This has become a common theme resulting in poor neonatal outcomes. The INFANT trial [3] actually concluded that CTG consistently detected abnormal FHR patterns but the clinicians failed to take appropriate decisions in time. Interestingly, when the cases of birth asphyxia are reviewed in retrospect during an investigation, clinical review or medicolegal proceedings, each case gets examined in isolation and it is often easy/natural to conclude in hindsight that appropriate action wasn't taken despite the "abnormal" CTG which is very likely to have been present. However, in reality the grass-root clinicians would have been looking after multiple cases with excessive (falsepositive) "abnormal" CTGs and had to select which ones to act upon based on flawed criteria. Confusion ensuing from a distorted framework and false alarms makes it difficult to "separate wheat from the chaff" thus increasing chance of erroneous decisions [24]. Thus, unconscious/unmindful to the birth attendants, an enforced wrong pattern-recognition (memeplex) of FHR decelerations seems to be seriously undermining their judgement/performance adversely affecting patient outcome and leading to high level of stress/repercussions for the staff [24]. An important scientific tenet is that, "the route from hypothesis (theory) to law (system/framework) to measurement (studies/trials) can almost never be travelled backwards" Thomas Kuhn, 1961. Thus no amount of systematic studies will prove, rectify or recompense frameworks based on false theories.

It is intuitive that the "total deceleration area" will have some correlation to incidence of fetal acidemia but it requires computerised measurement and reliable cut-offs have not been established or proven; and will need to be different for first and second stages of labor [14]. Secondly, smaller deceleration areas would be more significant in high risk cases (e.g. intrauterine growth retardation). Moreover, similar sizes of early and late decelerations will have very different significance. Thus, 
a purely mathematical algorithmic approach (even computerised) is unlikely to be the sole answer. An approach that incorporates both timing and size of the decelerations in the clinical contest is likely to give improved correlation to fetal acidemia necessary for clinical usefulness.

\section{Conclusions}

Recently, there has been a strong rhetoric that intrapartum CTG should be abandoned $[1,25]$. However, visual CTG interpretation will continue to be the most widely practiced method of intrapartum fetal monitoring for quite some time [24]. The FHR decelerations are the most important, complex and contested aspects of CTG interpretation. CTG as a science will be doomed without meaningful pattern-recognition of FHR decelerations $[9,24]$. Obstetricians and nurse-midwives need to critically analyse the three options of categorization of FHR decelerations, which are analogous to "memes" (ideas/narratives which evolve and replicate). The theory of "memetics" explains that the popular ideas may not necessarily be true (scientific) or without harm. It is a scientific imperative to cast off falsified theories (Sir Karl Popper). A pattern-recognition of FHR decelerations which is based on scientific hypotheses, careful observations and valid concepts should be chosen rather than the one that has simply become "popular" (like successful memes). The categorization of decelerations into early/ late/variable types based primarily on time relationship to contractions (similar to the British practice before 2007) seems to fulfil the scientific prerequisites $[9,11,12]$.

\section{Conflict of Interest}

The author has no conflict of interest to declare. The concepts presented are personal opinion only and do not necessarily reflect any practice. All the concepts were submitted to expertgroups on many occasions over the last decade.

\section{References}

1. Nelson KB, Sartwelle TP, Rouse DJ. Electronic fetal monitoring, cerebral palsy, and caesarean section: assumptions versus evidence. BMJ. 2016;355:16405.

2. Clark SL, Hamilton EF, Garite TJ, Timmins A, Warrick PA, Smith S. The limits of electronic fetal heart rate monitoring in the prevention of neonatal metabolic acidemia. Am J Obstet Gynecol. 2017;216(2):163 e161-163 e166.

3. The INFANT Collaborative Group. Computerised interpretation of fetal heart rate during labour (INFANT): a randomised controlled trial. Lancet. 2017;389(10080):17191729.

4. Royal College of Obstetricians and Gynaecologists. (2001) The use and interpretation of cardiotocography in intrapartum fetal surveillance. Evidence based Clinical Guideline No 8. London (UK): RCOG Press.

5. American College of Obstetricians and Gynecolo- gists. ACOG Practice Bulletin No. 106: Intrapartum fetal heart rate monitoring: nomenclature, interpretation, and general management principles. Obstet Gynecol. 2009;114(1):192-202.

6. National Institute for Health and Clinical Excellence (NICE). National Collaborating Centre for Women's and Children's Health (2014). Intrapartum care - Care of healthy women and their babies during childbirth. Guideline No 190, London (UK) [Cited 12 May 2017]. Available from URL: www.nice.org.uk/guidance/cg190.

7. Ayres-de-Campos D, Spong CY, Chandraharan E. FIGO consensus guidelines on intrapartum fetal monitoring: Cardiotocography. Int J Gynaecol Obstet. 2015;131(1):13-24.

8. Westgate JA, Wibbens B, Bennet L, Wassink G, Parer JT, Gunn AJ. The intrapartum deceleration in center stage: a physiologic approach to the interpretation of fetal heart rate changes in labor. Am J Obstet Gynecol. 2007;197(3):236 e231-211.

9. Sholapurkar SL. Critical imperative for the reform of British interpretation of fetal heart rate decelerations: analysis of FIGO and NICE Guidelines, Post-Truth Foundations, Cognitive Fallacies, Myths and Occam's Razor. J Clin Med Res. 2017;9(4):253-265.

10. Lear CA, Galinsky R, Wassink G, Yamaguchi K, Davidson JO, Westgate JA, Bennet L, et al. The myths and physiology surrounding intrapartum decelerations: the critical role of the peripheral chemoreflex. J Physiol. 2016;594(17):4711-4725.

11. Hon EH, Quilligan EJ. Electronic evaluation of fetal heart rate. IX. Further observations on "pathologic" fetal bradycardia. Clin Obstet Gynecol. 1968;11(1):145-167.

12. Mendez-Bauer C, Poseiro JJ, Arellano-Hernandez G, Zambrana MA, Caldeyro-Barcia R. Effects of atropine on the heart rate of the human fetus during labor. Am J Obstet Gynecol. 1963;85:1033-1053.

13. Memetics. Wikipedia, the free encyclopedia. https:// en.wikipedia.org/wiki/Memetics (Accessed April 2017).

14. Sholapurkar SL. Critical evaluation of American categorization of fetal heart rate (FHR) decelerations and three tier classification - Shortcomings, contradictions, remedies and need for debate. Open Journal of Obstetrics and Gynecology. 2013;3(3):362-370.

15. Cahill AG, Roehl KA, Odibo AO, Macones GA. Association and prediction of neonatal acidemia. Am J Obstet Gynecol. 2012;207(3):206 e201-208.

16. Sholapurkar SL. Categorization of Fetal Heart Rate Decelerations in American and European Practice: Importance and Imperative of Avoiding Framing and Confirmation Biases. J Clin Med Res. 2015;7(9):672-680.

17. Chung F, Hon EH. The electronic evaluation of fetal heart rate. I. With pressure on the fetal skull. Obstet Gynecol. 1959;13(6):633-640.

18. Giussani DA, Unno N, Jenkins SL, Wentworth RA, Derks JB, Collins JH, Nathanielsz PW. Dynamics of cardiovascular responses to repeated partial umbilical cord compression in late-gestation sheep fetus. Am J Physiol. 1997;273(5 Pt 2):H2351-2360.

19. Ugwumadu A. Are we (mis)guided by current guidelines 
on intrapartum fetal heart rate monitoring? Case for a more physiological approach to interpretation. BJOG. 2014;121(9):1063-1070.

20. Itskovitz J, LaGamma EF, Rudolph AM. Heart rate and blood pressure responses to umbilical cord compression in fetal lambs with special reference to the mechanism of variable deceleration. Am J Obstet Gynecol. 1983;147(4):451-457.

21. Lear CA, Galinsky R, Wassink G, Yamaguchi K, Davidson JO, Westgate JA, Bennet L, et al. Reply from Christopher A. Lear, Robert Galinsky, Guido Wassink, Kyohei Yamaguchi, Joanne O. Davidson, Jenny A. Westgate, Laura Bennet and Alistair J. Gunn. J Physiol. 2017;595(17):6081-6083.

22. MacLennan A. A template for defining a causal re- lation between acute intrapartum events and cerebral palsy: international consensus statement. BMJ. 1999;319(7216):1054-1059.

23. Heyborne KD. A systematic review of intrapartum fetal head compression: What is the impact on fetal brain? Am J Perinatol Rep. 2017;7(2):e79-e85.

24. Sholapurkar SL. Intrapartum cardiotocography remains valuable diagnostic tool, but FIGO/NICE may be inadvertently prescribing "wrong pattern-recognition". 10th December 2017. Response Re: Is continuous electronic fetal monitoring useful for all women in labour? http:// www.bmj.com/content/359/bmj.j5423/rr-2.

25. Mullins E, Lees C, Brocklehurst P. Is continuous electronic fetal monitoring useful for all women in labour? BMJ. 2017;359:j5423. 